\title{
The Prognosis for Control of Seizures with Medications in Patients with MRI Evidence for Mesial Temporal Sclerosis
}

\author{
Won-Joo Kim, Soo-Chul Park, Se-Jin Lee, Joon-Hong Lee, Jung-Yeon Kim, Byung-In Lee, and \\ *Dong-Ik Kim
}

Departments of Neurology and *Neuroradiology, College of Medicine, Yonsei University, Seoul, Korea

\begin{abstract}
Summary: Purpose: Mesial temporal sclerosis (MTS) is the most common and important pathology in temporal lobe epilepsy (TLE), and its presence in magnetic resonance imaging (MRI) scans is strongly correlated with a successful surgical outcome. Despite the general assumption that patients with MTS respond poorly to medication, the long-term prognosis for such patients has not yet been investigated. We studied the overall clinical prognosis of patients with MTS and analyzed the factors related to the degree of medical responsiveness.

Methods: Case patients were actively followed up at the Yonsei Epilepsy Clinic in Seoul, Korea, for $>2$ years. A structured interview and a thorough clinical evaluation were conducted. MRI scans, at the field strength of 1.0 or 1.5 Tesla, were performed with $T_{1}$ - and $T_{2}$-weighted coronal and axial spin-echo images. All coronal slices were $\leqslant 5-\mathrm{mm}$ thick with no gap. Two neurologists and one radiologist determined the presence of MTS in MRIs by visual analysis.
\end{abstract}

Results: The MTS group consisted of 104 patients. Of these,
$26(25 \%)$ were completely controlled with adequate therapy, and $40(38 \%)$ were intractable, despite aggressive anticonvulsant polytherapy. The remaining $37 \%$ had their seizure frequencies reduced by $\geqslant 50 \%$, but were not seizure free. The age of seizure onset was significantly younger in the intractable group than in the well-controlled group. Patients with a history of febrile convulsions or with epileptiform discharges in their electroencephalogram (EEG) had poorer seizure control $(\mathrm{p}<$ 0.05 ) than those who did not. Among the 16 patients who had no previous treatment, five $(31 \%)$ became seizure free, and two were intractable.

Conclusions: Not all patients with MTS are medically intractable; $25 \%$ of the patients in our study achieved complete control while receiving medication. Poor seizure control was related to an early age of seizure onset, a history of febrile convulsions, and epileptiform discharges on the EEG. Key Words: Mesial temporal sclerosis-MRI-SeizureEpilepsy-Prognosis.
Hippocampal sclerosis is the most common pathologic finding in in temporal lobe epilepsy (TLE). It is found in $50-70 \%$ of cases, after temporal lobectomy for intractable temporal lobe epilepsy (1).

Magnetic resonance imaging (MRI) is the most valuable imaging technique for epilepsy. It can more accurately evaluate mass and other structural lesions of the temporal lobe than computed tomography (CT) $(2-4)$. The MRI abnormalities of hippocampal sclerosis-an increased hippocampal signal on $T_{2}$-weighted images, a decreased signal on $T_{1}$-weighted images, and hippocampal atrophy-have been extensively described, and the presence of these features on MRI have enabled physicians to diagnose hippocampal sclerosis in $80-90 \%$ of cases (5). These features also indicate mesial temporal sclerosis (MTS). Therefore it is possible to recognize

Accepted July 27, 1998

Address correspondence and reprint requests to Dr. W. Kim at Department of Neurology, Yongdong Severance Hospital, 146-92 Dogok-dong Kangnam-gu, Seoul, Korea.
MTS without surgical exposure. In this study, we assessed the prognosis for seizure control of patients whose MTS was found through MRI and determined factors influencing the course of their disease and responsiveness to medical treatment.

\section{METHODS}

We evaluated MRI scans from 104 patients at the Yonsei Epilepsy Program in Seoul, Korea, from January 1989 to September 1996. The patients were referred there for expert consultation or came on their own. We first selected a patient group from those with clinical features of complex partial seizures or who had a temporal focus on interictal electroencepalograms (EEGs). We excluded patients who had an aura-visual, simple motor, or simple sensory-that had a non-temporal lobe origin. Patients who had had loss of consciousness or nausea and vomiting after a head injury were considered to have experienced head trauma.

Two kinds of MRI machines were used. One was a 
1.5-Telsa magnet (Signa, General Electric, Milwaukee, WI, U.S.A.) and the other was a 1.0-Telsa magnet (Magnetom Impact, Siemens, Danvers, MA, U.S.A.). $\mathrm{T}_{2}-$ weighted images were spin-echo sequences, with echo times (TE) of 80 and $85 \mathrm{~ms}$ and repetition time (TR) of 2,200 and 4,500 $\mathrm{ms}$. $\mathrm{T}_{1}$-weighted images were TE 15-ms and TR $600-\mathrm{ms}$ sequences. We used only $\mathrm{T}_{1}$ and $\mathrm{T}_{2}$ signal images without inversion recovery since an inversion recovery method has been part of our MRI protocol since 1997.

We performed axial views parallel to the long axis of the hippocampus. In coronal views, we transected the long axis of the hippocampus to evaluate its oval-shaped appearance. All slices were 5- or 3-mm thick with a no-gap method.

Three observers (two epileptologists and one neuroradiologist) evaluated the MRIs independently, without prior access to information about the patients or each other's opinions. Each observer was asked to make an overall diagnosis and to confirm the lateralization of the lesion. They classified the scans as normal or as MTS. The case patients for this study were chosen if at least two observers diagnosed MTS. We excluded patients with dual abnormal findings, such as temporal lobe atrophy, vascular malformations, cerebral infarction, tumor, and cortical dysplasia of the temporal lobe. We also excluded those suspected of having bilateral hippocampal sclerosis and those with a loss of internal morphologic structure because of visual uncertainty.

The EEG was performed for about 40 minutes by the routine method with nasopharyngeal electrodes. An abnormal EEG was defined as epileptiform discharges around the temporal lobe.

The patients were followed up for more than 2 years. The average seizure frequency during the second year was used for evaluating seizure frequency, because the frequency in the first year was subject to change due to adjustments in medication. The initial seizure frequency was determined by historical reviews; during the followup period, we obtained seizure frequencies from the seizure diaries of the patients.

We divided the patients into three groups according to their outcome after 2 years of follow-up. The first group consisted of patients who were seizure free during the second year of follow-up. The second group consisted of patients with improved seizure control whose seizure frequency had decreased $>50 \%$ from their initial rate. The third group consisted of patients with poor seizure control whose seizure frequency had decreased $<50 \%$ or had not changed from their initial frequency.

We also asked the patients about their antiepileptic drug (AED) history to determine if they had been treated adequately before visiting our epilepsy clinic. If the serum $A E D$ levels were in the therapeutic range at their first visit, they were considered to have been adequately treated.

\section{RESULTS}

The average age of patients at onset of seizures was $14.6 \pm 7.3$ years (range, $3-35$ years), and the average duration of illness was $13.1 \pm 7.9$ years. There were 60 men $(57 \%)$ and 44 women patients $(43 \%)$. Fifty-three patients (51\%) had MTS in the left side and 51 (49\%) had it in the right. No significant correlation was found between the location of the lesion, either in the right or left side, and seizure outcome, nor was there a difference in seizure outcome between the men and women (Table 1).

Thirty-seven patients (35\%) had experienced febrile convulsions; 20 (19\%) had experienced meningoencephalitis; and $10(10 \%)$ had experienced head trauma.

According to the criteria of the follow-up outcome, 26 (25\%) patients belonged to the seizure-free group, 38 $(37 \%)$ to the improved-seizure-control group, and 40 (38\%) to the poor-seizure-control group.

The onset age was $16.9 \pm 8.5$ years for the seizure-free group, $15.9 \pm 6.9$ years for the improved-seizure-control group, and $13.0 \pm 5.9$ years for the poor-seizure-control group ( $\mathrm{p}<0.05$ ). The duration of illness in each group was not significantly different: $12.8 \pm 6.9$ years, $13.7 \pm$ 8.9 years, and $12.4 \pm 8.2$ years, respectively.

The median seizure frequency per month during the follow-up period of the improved-seizure-control group was three (IQ range, 1-7), compared with five for the poor-seizure-control group (range, 1-18).

Based on the routine EEGs that were taken during each patient's first visit, $14(40 \%)$ with normal EEGs became seizure free, whereas only $12(18 \%)$ of those with focal epileptiform discharges did $\left(\mathrm{p}<0.05, \chi^{2}=\right.$ 5.33).

Thirty percent of the MTS patients in our study had experienced febrile convulsions. Patients with a history of a febrile convulsion $(49 \%)$ had worse seizure control than patients who had no definite history $(28 \%, \mathrm{p}<0.01$, $\left.\chi^{2}=8.34\right)$. Other events such as central nervous system (CNS) infection, head trauma, and hypoxia were not correlated with prognosis for seizure control.

Of the patients previously treated before referral to our center, those who had been treated adequately were less likely to achieve improved seizure control than those who had been inadequately treated $\left(p<0.05, \chi^{2}=8.47\right)$. After the 2-year follow-up period, no patients receiving three or more AEDs were seizure free, whereas 21 patients with monotherapy (43\%) were.

Sixteen patients had not taken any AEDs before visiting our clinic. All were started with AED treatment. Of those, five $(31 \%)$ were seizure free after 1 year of treatment with monotherapy, nine $(56 \%)$ were improved, and two $(13 \%)$ were poorly improved with medications. 
TABLE 1. Clinical data of patients

\begin{tabular}{|c|c|c|c|}
\hline Characteristics & $\begin{array}{c}\text { Seizure free } \\
(\mathrm{n}=26) \\
(\%)\end{array}$ & $\begin{array}{l}\text { Improved control } \\
(\mathrm{n}=38) \\
(\%)\end{array}$ & $\begin{array}{l}\text { Poor control } \\
(\mathrm{n}=40) \\
(\%)\end{array}$ \\
\hline Age of onset $(\mathrm{yr})^{a}$ & $16.9 \pm 8.5$ & $15.9 \pm 6.9$ & $13.0 \pm 5.9$ \\
\hline Duration of illness (yг) & $12.8 \pm 6.9$ & $13.7 \pm 8.9$ & $12.4 \pm 8.2$ \\
\hline \multicolumn{4}{|l|}{ Lesion side } \\
\hline Left & $13(25)$ & $23(43)$ & $17(32)$ \\
\hline Right & $13(25)$ & $15(29)$ & $23(46)$ \\
\hline \multicolumn{4}{|l|}{ Sex } \\
\hline Male & $14(23)$ & $21(35)$ & $25(42)$ \\
\hline Female & $12(27)$ & $17(39)$ & $15(34)$ \\
\hline \multicolumn{4}{|l|}{$\mathrm{EEG}^{\alpha}$} \\
\hline Normal & $14(40)$ & $11(31)$ & $10(29)$ \\
\hline Abnormal & $12(18)$ & $27(40)$ & $29(42)$ \\
\hline \multicolumn{4}{|l|}{ Risk factors in history } \\
\hline None & $11(38)$ & $10(34)$ & $8(28)$ \\
\hline Febrile convulsions ${ }^{a}$ & $3(8)$ & $16(43)$ & $18(49)$ \\
\hline CNS infection & $6(30)$ & $6(30)$ & $8(40)$ \\
\hline Head trauma & $3(30)$ & $3(30)$ & $4(40)$ \\
\hline Others (hypoxia, etc.) & $3(38)$ & $3(38)$ & $2(24)$ \\
\hline \multicolumn{4}{|l|}{ Treatment history } \\
\hline Not treated & $5(31)$ & $9(56)$ & $2(13)$ \\
\hline Inadequately treated & $14(34)$ & $16(39)$ & $11(27)$ \\
\hline Adequately treated & $7(15)$ & $13(28)$ & $26(57)$ \\
\hline \multicolumn{4}{|l|}{ Antiepileptic drug usage } \\
\hline Monotherapy & $21(43)$ & $24(49)$ & $4(8)$ \\
\hline Polytherapy with 2 drugs & $5(14)$ & $13(36)$ & $18(50)$ \\
\hline Polytherapy with 3 or more drugs & $0(0)$ & $1(5)$ & $18(95)$ \\
\hline
\end{tabular}

"Significance between seizure-free group and poor-seizure-control group $(p<0.05)$.

\section{DISCUSSION}

Temporal lobe epilepsy is the most common cause of complex partial seizures. Any kind of lesion in the temporal lobe can result in TLE. By the site of a lesion, we usually classify a disorder as mesial TLE or lateral TLE. Lateral TLE is caused by various lesions, but mesial TLE has a relatively homogeneous, pathologic substrate, which can often be treated surgically. The term "mesial temporal lobe epilepsy" is considered a unique entity of TLE. The term "mesial temporal sclerosis" was introduced by Falconer and Taylor (6).

The pathologic term "hippocampal sclerosis (HS)' is common in the autopsies of patients with epilepsy and includes neuronal loss and secondary glial fibrosis $(1,7)$. Until recently, no diagnostic imaging tool, not even CT, could evaluate MTS. Advances made in MRI techniques have made the identification of small mesial temporal structures possible. Cell loss, if sufficient, will appear in the MRI scan as changes in signal intensity and volume loss $(8,9)$. The basic features of MRI change in MTS that we found were a decreased signal and atrophic change in $\mathrm{T}_{1}$-weighted images and an increased signal in $\mathrm{T}_{2}$ images $(10,11)$. Other helpful features were asymmetry of the temporal horns and enlarged sylvian fissures in the ventral orientation. In $\mathrm{T}_{2}$-weighted images, we attempted to visualize the cerebrospinal fluid around the temporal horn and the choroidal fissures (11). To evaluate MTS, MRI scans interpreted by visual inspection alone had a lower determination value than volumetric estimations of the hippocampus (12), but when hippocampal volume ratios were $<0.7$, visual detection was as accurate as that of volumetrics (13).

An early age of onset is one of the poor prognostic factors of TLE. Lindsay, et al. (14) found that patients whose onset of seizures was before 2 years of age had a bad prognosis. (In children under age 15 years with temporal epilepsy, the age at onset was considered the age of the first seizure, whatever its nature.) Fois, et al. (15) found that the unresponsive group in their study had an earlier mean age of onset than the responsive group. The results of this study also showed that an early onset age affected the outcome more than the duration of the illness, and that a significant inversion correlation existed between the age of onset and the grade of HS (16).

Focal epileptiform discharges in anterior and midtemporal regions predicted intractability to medication (15). Patients with normal EEG features had a better prognosis than those with abnormal ones.

A previous study found that the side of focus in an EEG predicted the prognosis, with the left side showing poorer results (14). We considered the MTS side as the lesion side, but could not find any significant difference between the right and the left.

A relatively high percentage of the patients with MTS who were candidates for surgical treatment or for TLE had a history of febrile convulsions $(17-20)$. There is much speculation regarding the relation between febrile 
convulsions and MTS. One theory is that MTS is caused by a factor independent of the epilepsy, another that MTS is caused as a consequence of epilepsy. Murakami et al. (21) found that $11(58 \%)$ of their 19 patients had febrile convulsions, and that among them, 7 (37\%) had prolonged ones. Reports have shown that prolonged febrile convulsions play an etiologic role in the development of MTS, that they correlate positively with hippocampal atrophy, and are related to the duration of illness (22). Our results also showed that the patients with a history of febrile convulsions have a poorer prognosis than those without. No significant correlation between the prognosis and the duration of illness was noted.

The dosage of AEDs may be another important consideration for patients. New patients visiting our clinic are usually referral cases. Of the case patients, 85 were referred because their epilepsy had not improved, even with medication. Judging from the serum drug level at their first visit, 41 patients had been given an insufficient dosage. Almost 30 patients showed improvement or were seizure free after dosage adjustment or drug change. When dosages were adjusted, many intractable patients improved; however, no patients receiving three or more drugs were seizure free after a 2-year follow-up period. Because the seizure-free rate after temporal lobectomy of MTS is $-70 \%$, patients whose seizures are not well controlled with two drugs should be considered for surgery rather than prescribed additional drugs (23).

Many surgical reports have shown that a large proportion of temporal lobectomy patients had pathologic ab normalities, especially HC. In a study of newly diagnosed seizure patients, those with hippocampal sclerosis had a worse prognosis than those with other MRI findings (24), and Murakami et al. (21) found that only two out of their 19 MTS patients were controlled with medication. We may assume from these results that seizures of patients with HS are intractable and need surgical treatment. But we found that $25 \%$ of our patients became seizure free while receiving medication. Those with no previous treatment were few, but five such patients became seizure free, and $56 \%$ improved with better medical treatment. These results suggest that MTS patients are not always resistant to medical treatment, and 2-year trials of AED therapy may be appropriate. But patients whose seizures are only poorly controlled with three or more drugs should be considered for possible surgery without further delay.

\section{REFERENCES}

1. Margerison JH, Corsellis JAN. Epilepsy and temporal lobes: clinical, electroencephalographic and neuropathological study of the brain in epilepsy, with particular reference to the temporal lobes. Brain 1966;89:499-530.
2. Kuznieky R, de la Sayette V, Ethier R, et al. Magnetic resonance imaging in temporal lobe epilepsy: pathological correlations. Ann Neurol 1987;22:341-7.

3. Theodore WH, Katz D, Kufta C, et al. Pathology of temporal lobe foci: correlation with CT, MRI and PET. Neurology 1990;40:797803.

4. Dowd CF, Dillon WP, Barbaro NM, Laxer KD. Magnetic resonance jmaging of intractable complex partial seizures: pathologic and electroencephalographic correlation. Epilepsia 1991;32:4549.

5. Jackson GD, Berkovic SF, Duncan JS, Connelly A. Optimizing the diagnosis of hippocampal sclerosis using MR imaging. AJNR 1993:14:753-62

6. Falconer MA, Taylor DC. Surgical treatment of drug-resistant epilepsy due to mesial temporal sclerosis. Arch Neurol 1968;19:35361 .

7. Meencke HJ, Veith G. Hippocampal sclerosis in epilepsy. In: Luders HO, ed. Epilepsy surgery. New York: Raven Press, 1991:70515.

8. Jack CR, Sharbrough FW, Twomey CK, et al. Temporal lobe seizures: lateralization with MR volume measurements of the hippocampal formation. Radiology 1990;175:423-9.

9. Sperling MR, Wilson G, Engel J, Babb TL, Phelps M, Bradley W. Magnetic resonance imaging in intractable partial epilepsy: correlative studies. Ann Neurol 1986;20:57-62.

10. Duncan JS. Imaging and epilepsy. Brain 1997;120:339-77.

11. Jackson GD, Berkovic SF, Tress BM, Kalnins RM, Fabinyi GCA, Baldin PF. Hippocampal sclerosis can be reliably detected by magnetic resonance imaging. Neurology 1990;40:1869-75.

12. Garcia PA. Laxer KD, Barbaro NM. Dillon WP. Prognostic value of qualitative magnetic resonance imaging hippocampal abnormalities in patients undergoing temporal lobectomy for medically refractory seizures. Epilepsia 1994;35:520-4.

13. Reutens DC, Stevens JM, Kingsley D, et al. Reliability of visual inspection for detection of volumetric hippocampal asymmetry. Neuroradiology 1996:38:221-5.

14. Lindsay J, Qunsted C, Richard P. Long-term outcome in children with temporal lobe seizures: social outcome and childhood factors. Dev Med Child Neurol 1979;21:285-98.

15. Fois A, Tomaccini D, Balestri P, Malandrini F, Vascotto M, DeFeo F. Intractable epilepsy: etiology, risk factors and treatment. Clin Electroencephalogr 1988;19:68-73.

16. Davies KG, Hermann BP, Dohan FC Jr, Foley KT, Bush AJ, Wyler AR. Relationship of hippocampal sclerosis to duration and age of onset of epilepsy, and childhood febrile seizures in temporal lobectomy patients. Epilepsy Res 1996;24:119-26.

17. French JA, Williamson PD, Thadani VM, et al. Characteristics of mesial temporal lobe epilepsy: results of history and physical examination. Ann Neurol 1993:34:774-80.

18. Cendes F, Andermann F, Dubeau F, et al. Early childhood prolonged febrile convulsion, atrophy and sclerosis of mesial structures and temporal lobe epilepsy: an MRI volumetric study. Neurology $1993 ; 43: 1083-7$

19. Sagar HJ, Oxbury JM. Hippocampal neuron loss in temporal lobe epilepsy: correlation with early childhood convulsion. Ann Neurol 1991:30:31-6.

20. Jensen I. Temporal lobe epilepsy: etiological factors and surgical results. Acta Neurol Scand 1976;53:103-18.

21. Murakami N, Ohno S, Oka E, Tanaka A. Mesial temporal lobe epilepsy in childhood. Epilepsia 1996;37(suppl 3):52-6.

22. Cendes F, Andermann F, Gloor P, et al. Atrophy of mesial structures in patients with temporal lobe epilepsy: cause or consequence of repeated seizures? Ann Neurol 1993;34:795-801.

23. Engel J Jr, Van Ness PC, Rasmussen TB, Ojemann LM. Ontcome with respect to epileptic seizures. In: Engel J Jr, ed. Surgical treat ment of the epilepsies. New York: Raven Press, 1993:609-21.

24. Van Paesschen W, Duncan JS, Stevens JM, Connelly A. Etiology and early prognosis of newly diagnosed partial seizures in adults: a quantitative hippocampal MRI study. Neurology 1997;49:753-7. 